\title{
Positive feedback regulation between Akt phosphorylation and fatty acid synthase expression in osteosarcoma
}

\author{
HENG WANG ${ }^{1 *}$, QING FENG LUO ${ }^{2 *}$, AI FEN PENG ${ }^{3}$, XIN HUA LONG $^{1}$, TAO FANG WANG ${ }^{1}$, ZHI LI LIU $^{1}$, \\ GUO MEI ZHANG ${ }^{4}$, RONG PING ZHOU ${ }^{4}$, SONG GAO ${ }^{1}$, YANG ZHOU ${ }^{1}$ and WEN ZHAO CHEN ${ }^{1}$ \\ ${ }^{1}$ Department of Orthopedics, First Affiliated Hospital of Nanchang University; ${ }^{2}$ Department of Pathology, \\ Cancer Hospital of Jiangxi Province; ${ }^{3}$ Jiangxi University of Traditional Chinese Medicine; ${ }^{4}$ Department of Orthopedics, \\ Second Affiliated Hospital of Nanchang University, Nanchang, Jiangxi, P.R. China
}

Received September 2, 2013; Accepted December 13, 2013

DOI: $10.3892 /$ ijmm.2013.1602

\begin{abstract}
The activation of PI3K/Akt and the overexpression of fatty acid synthase (FASN) are frequently observed in human osteosarcoma (OS). In the present study, in order to investigate the possible association between the phosphorylation of Akt and FASN expression, immunohistochemical staining was conducted on 24 OS specimens from patients with pulmonary metastasis, which revealed a significant positive correlation between phosphorylated Akt (p-Akt) and the expression of FASN $(\mathrm{R}=0.469, \mathrm{P}=0.04)$. To investigate the association between $\mathrm{p}$-Akt and FASN in vitro, human U2-OS OS cells were treated with FASN-specific RNAi plasmid or LY294002 (an inhibitor of PI3k/Akt). The mRNA levels of Akt and FASN were measured by real-time PCR. Western blot analysis was also performed to detect the protein experession of PI3K, Akt, p-Akt and FASN. The results demonstrated that the PI3K/Akt signaling pathway modulates FASN expression; the inhibition of FASN resulted in the downregulation of p-Akt in the U2-OS cells. Furthermore, the effects induced by the inhibition of the activity of p-Akt or FASN on the malignant phenotype of U2-OS cells were investigated, demonstrating that the malignant phenotype was inhibited by suppressing the activity of PI3K/Akt or FASN in the U2-OS cells. The findings from our study suggest the existence of a positive feedback regulation between Akt phosphorylation and FASN expression and that this loop may play an important role in the malignant phenotype of OS cells.
\end{abstract}

Correspondence to: Professor Zhi Li Liu, Department of Orthopedics, First Affiliated Hospital of Nanchang University, 17 Yong Wai Zheng Street, Nanchang, Jiangxi 330006, P.R. China E-mail: zgm7977@163.com

*Contributed equally

Key words: osteosarcoma, metastasis, fatty acid synthase, PI3K/ Akt signaling pathway

\section{Introduction}

Osteosarcoma (OS) is one of the most common primary malignant bone tumors in children and adolescents. Following the advent of effective chemotherapy, the five-year survival rate for patients with OS treated with intensive multidrug chemotherapy and aggressive local control has been reported to be $55-80 \%$ (1-3). However, numerous studies have reported that the five-year survival rate of patients with metastatic diseases is $<20 \%$ (4-6). The development of lung metastasis is the main cause of mortatlity in patients with OS. The identification and understanding of the molecular mechanisms responsible for metastasis would pose a significant impact on the management of OS.

Fatty acid synthase (FASN) is an enzyme which is considered crucial for endogenous lipogenesis in mammals, and is responsible for catalyzing the synthesis of long-chain fatty acids. In the majority of normal cells, FASN expression is usually not observed, due to the presence of abundant amounts of dietary lipids (7). However, FASN is overexpressed in a variety of human tumors (8-12), and has been strongly linked to cancer cell proliferation and apoptosis (13-16). We previously demonstrated that FASN may contribute to the metastasis of OS cells $(17,18)$. However, its potential molecular mechanisms of action remain unclear.

PI3K/Akt plays a crucial role in the cell-extracellar matrix (ECM) and cell-cell adhesion. Due to lack of correct adhesion, the adhesion-dependent signals are interrupted, resulting in adhesion-related apoptosis, namely anoikis. PI3K/Akt signaling has been implicated in the regulation of FASN expression in breast cancer and prostate cancer cells $(19,20)$. Wang et al reported that there was a positive feedback regulation between Akt phosphorylation and FASN expression in ovarian carcinoma cells (21). However, to our knowledge, the association between the phosphorylation of Akt and FASN protein expression in OS has not yet been documented.

In this study, we found that the inhibition of Akt phosphorylation by LY294002 (an inhibitor of PI3k/Akt), resulted in the downregulation of FASN expression. In addition, the downregulation of FASN expression inhibited Akt phosphorylation. Based on these findings, we confirmed the existence of a positive feedback loop between Akt phosphorylation and 
FASN expression; this feedback loop may play an important role in the malignant phenotype of OS cells.

\section{Materials and methods}

Patient specimens. A total of 24 samples of OS tissues were obtained from patients with pulmonary metastatic disease who underwent surgery in our hospital (The First Hospital Affiliated to Nanchang University, Nanchang, China) from 2005 to 2012. The pulmonary metastasis survey was performed with plain films and chest CT scans at first diagnosis. All the patients had no history of prior treatment with anticancer drugs or radiotherapy. The samples were fixed with $10 \%$ formalin and embedded in paraffin and were then cut into $4-\mu \mathrm{m}$-thick sections. In all cases, informed consent was obtained from the relative departments and persons, and the study had the approval of the Ethics Committee of Nanchang University.

Immunohistochemistry. Immunohistochemical (S-P) staining with and hematoxylin and eosin (H\&E) was performed on the paraffin-embedded tissue sections. Antigen retrieval was performed by heating the sections in $10 \mathrm{mmol} / 1$ citrate buffer (pH 6.0) for $20 \mathrm{~min}$. FASN and phosphorylated Akt (p-Akt) antibodies (rabbit monoclonal antibody; antibody dilutions, 1:50; Epitomics, Inc., Burlingame, CA, USA) were used as the primary antibody at a final dilution as corresponding product specifications. The sections were then stained with diaminobenzidine (DAB) and counterstained using hematoxylin. The stained sections were evaluated and scored by two pathologists in a blinded manner without prior knowledge of the clinical pathological characteristics of the patients. According to the staining intensity by examining at least 500 cells in five representative areas, the expression level of p-Akt and FASN was measured and the intensity scores were recorded as follows: none, 0 ; weak, 1 ; moderate, 2; and intense, 3 . According to the percentage of cancer cells with a positive expression of Akt and FASN, the percentage scores were recorded as follows: $0 \%$ (score 0 ); $<10 \%$ (score 1 ); $10-49 \%$ (score 2); 50-79\% (score 3); and 80-100\% (score 4). The final score was averaged with the scores from the two pathologists; these scores were calculated by multiplying the intensity score by the percentage score. The sections with a final score of $<4$ were considered as negative (-), those with a score of 4-5 were considered as postivie $(+)$, those with a score of 6-8 as double positive (++), and those with a score of 9-12 were considered as triple positive $(+++)$.

Cell culture and transfection. The human OS cell line, U2-OS, was purchased from the American Type Culture Collection (ATCC; Manassas, VA, USA), and the cells were routinely cultured in RPMI-1640 medium (HyClone, Logan, UT, USA) supplemented with $10 \%$ fetal bovine serum (FBS) (Sigma, Lenexa, KS, USA) in a humidified $37^{\circ} \mathrm{C}$ incubator containing $5 \% \mathrm{CO}_{2}$. The U2-OS cells were seeded in 6-well plates till $40 \%$ confluence on the day prior to transfection. The U2-OS cells were transfected with FASN-specific RNAi plasmid (MR-FASN) and the negative control RNAi plasmid (MR-Neg) using Lipofectamine 2000 according to the instructions of the manufacturer (Invitrogen Life Technologies (Carlsbad, CA, USA).
Table I. Primer sequences of genes used in real-time PCR.

Gene (size, bp) Primer sequence $\left(5^{\prime} \rightarrow 3^{\prime}\right)$

\begin{tabular}{ll}
\hline FASN (171) & F AACTCCATGTTTGGTGTTTG \\
& R CACATGCGGTTTAATTGTG \\
Akt (198) & F TGCCACCATGAATGAGGTGAAT \\
& R GCGTATGACAAAGGTGTTGGG \\
GAPDH (199) & F CAGGGCTGCTTTTAACTCTGGT \\
& R GATTTTGGAGGGATCTCGCT
\end{tabular}

FASN, fatty acid synthase; GAPDH, glyceraldehyde-3-phosphate dehydrogenase; $\mathrm{F}$, forward, $\mathrm{R}$, reverse.

Real-time PCR. Semi-quantitive (real-time) PCR was used to detect the FASN mRNA expression levels. Total RNA was extracted from the cells using TRIzol reagent (Invitrogen Life Technologies). The total RNA concentration was determined by spectrophotometry at $260 \mathrm{~nm}$ and the purity was determined by calculating the $260 / 280$ ratio with a BioPhotometer (Eppendorf, Hamburg, Germany). The two-step kit (Promega Corp., Madison, WI, USA) was used to to obtain cDNA according to the manufacturer's instructions, which was then used as the template for amplification. Glyceraldehyde-3phosphate dehydrogenase (GAPDH) was used as an internal standard. The gene primer sequences are listed in Table I. The cycling conditions were as follows: initial denaturation at $94^{\circ} \mathrm{C}$ for $3 \mathrm{~min}$, followed by 35 amplification cycles of $94^{\circ} \mathrm{C}$ for $30 \mathrm{sec}, 55^{\circ} \mathrm{C}$ for $30 \mathrm{sec}$ and $72^{\circ} \mathrm{C}$ for $12 \mathrm{sec}$. Each real-time PCR assay contained $2 \mu \mathrm{l}$ cDNA template, $10 \mu \mathrm{l}$ SuperMix and $0.5 \mu \mathrm{l}$ of every forward and reverse primer in a $20 \mu \mathrm{l}$ reaction mixture. All experiments were repeated six times over multiple days.

Western blot analysis of protein expression. Total protein from the cells was extracted using RIPA lysis buffer containing $60 \mu \mathrm{g} / \mathrm{ml}$ phenylmethylsulfonyl fluoride (PMSF). The protein concentrations were determined using the BCA protein assay kit (Boster Biotechnology Co., Wuhan, China). The protein samples were denatured at $100^{\circ} \mathrm{C}$ for $10 \mathrm{~min}$ and then preserved at $-20^{\circ} \mathrm{C}$ for later use. The proteins were separated by SDS-polyacrylamide gel electrophoresis and transblotted onto PVDF membranes. The PVDF membranes were probed first with primary antibodies PI3K, Akt and p-Akt (Ser473) antibody (1:1,000 dilution), FASN antibody (1:500 dilution) (both from Santa Cruz Biotechnology, Inc., Santa Cruz, CA, USA)and $\beta$-actin antibody (1:2,000; Cell Signaling Technology, Inc., Danvers, MA, USA) overnight at $4^{\circ} \mathrm{C}$. Following incubation with the appropriate anti-rabbit or anti-mouse horseradish peroxidase-conjugated secondary antibody (1:5,000; Boster Biotechnology Co.) for $1.5 \mathrm{~h}$ at room temperature, immunoreactive bands were visualized by chemiluminescence dissolvent (Thermo Scientific, Rockford, IL, USA) and exposured to X-ray film (Kodak, Rochester, NY, USA). The determination of the grayscale value was processed using ImageJ sofware. All experiments were repeated six times over multiple days. 


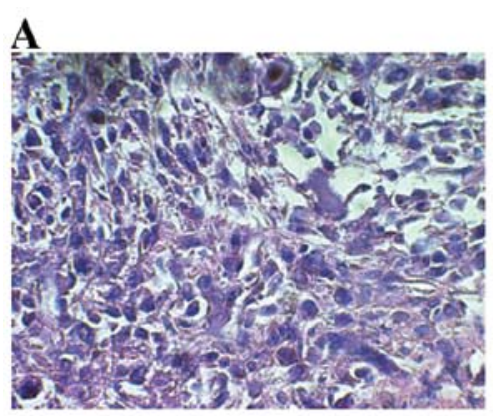

$\mathrm{H} \& \mathrm{E}(\mathrm{x} 400)$

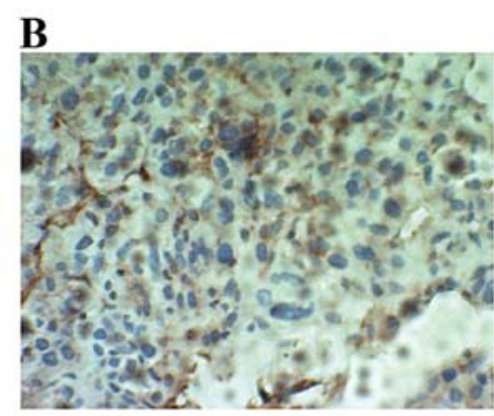

FASN $(x 400)$

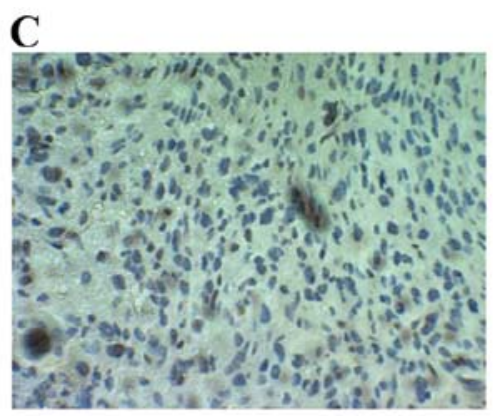

p-Akt (x400)

Figure 1. Immunohistochemical staining used to examine fatty acid synthase (FASN) and phosphorylated Akt (p-Akt) (Ser473) expression. (A) Hematoxylin and eosin (H\&E) staining in osteosarcoma (OS) tissues from patients with pulmonary metastasis (x400). (B) FASN protein is shown as brownish-yellow particle deposition and is expressed in the cytoplasm. (C) p-AKT is shown as brownish-yellow particle deposition and is expressed in the cytoplasm and nucleus.

Cell proliferation assay. Cells $\left(4 \times 10^{3} / 200 \mu 1 /\right.$ well $)$ were seeded in 96-well plates. Viable proliferating cells were detected by 3-(4,-dimethy-lthiazol-2-yl)-2,-diphenyl-tetrazoliumbromide (MTT) assay at various time periods (24, 48 and $72 \mathrm{~h}$ ), using five wells per time period. Cell viability was expressed as the optical density (OD), which was detected by an enzyme-linked immunoabsorbent assay reader (MK3; Thermo Scientific) at a 490-nm wavelength. All experiments were repeated six times over multiple days.

Analysis of cell apoptosis. Cells $\left(5 \times 10^{5}\right)$ were harvested, washed with PBS and resuspended in binding buffer, followed by mixing with Annexin V-FITC and propidium iodide (both from KeyGen Biotech. Co., Ltd., Nanjing, China). The cells were analyzed by a BD FACSCalibur flow cytometer (BD Biosciences, San Jose, CA, USA). All experiments were repeated six times over multiple days.

Transwell invasion assays. Cell invasion was measured in 24-well plates by Transwell assay using a chamber containing the polyethylene terephthalate filter membrane with $8-\mu \mathrm{m}$ pores (BD Biosciences). The cells $\left(6 \times 10^{4} / 200 \mu 1 /\right.$ chamber) were seeded in the upper chamber with RPMI-1640 medium containing $10 \mathrm{~g} / \mathrm{l} \mathrm{BSA}$, and the lower well was filled with $500 \mu$ l RPMI-1640 medium supplemented with $10 \%$ FBS as a chemoattractant. Following incubation for $24 \mathrm{~h}$, the chambers were stained with crystal violet. The invaded cells were counted from ten randomly selected fields under an inverted microscope. All experiments were repeated six times over multiple days.

Wound healing assays. Cell migration was assessed by determining the ability of the cells to move into a cellular space in a two-dimensional in vitro 'wound healing assay'. In brief, the cells were grown to confluence in 6-well tissue culture plastic dishes to a density of $5 \times 10^{6}$ cells/well. The cells were denuded by dragging a rubber policeman (Fisher Scientific, Hampton, $\mathrm{NH}, \mathrm{USA}$ ) through the center of the plate. The cultures were rinsed with PBS and replaced with fresh DMEM alone or containing $10 \% \mathrm{FBS}$, following which the cells were incubated at $37^{\circ} \mathrm{C}$ for $24 \mathrm{~h}$. Images were captured at 0 and $24 \mathrm{~h}$ and the migrated distance was measured using ImageJ software (NIH,
Bethesda, MD, USA). All experiments were repeated six times over multiple days .

Statistical analysis. Statistical comparisons were performed using SPSS software version 13.0 (SPSS Inc., Chicago, IL, USA). The correlation of FASN with p-Akt protein in the OS tissues was evaluated using the Wilcoxon rank sum test. All measurement data are presented as the means $\pm \mathrm{SD}$, and the one-way ANOVA with a post-hoc test (Student-Newman-Keuls test) was performed for statistical analysis. A P-value $<0.05$ was considered to indicate a statistically significant difference.

\section{Results}

Correlation between FASN and p-Akt protein expression in $O S$. To explore the possible association between FASN expression and the phosphorylation of Akt in OS, FASN and p-Akt (Ser473) protein expression in the OS tissues from patients with pulmonary metastatic disease was detected. The results revealed that FASN protein was expressed in the cytoplasm of the OS tissues, and the p-Akt protein was expressed in the nucleus and cytoplasm (Fig. 1A-C). There was a significant positive relationship between FASN and p-Akt expression $(\mathrm{R}=0.469, \mathrm{P}=0.04)$. These data suggest that a possible connection between FASN expression and the phosphorylation of Akt exists in OS.

Inhibiting the phosphorylation of Akt suppresses the malignant phenotype of U2-OS cells. To determine the effects of the inhibition of Akt phosphorylation on the malignant phenotype of OS cells, the U2-OS cells were treated with LY294002 (an inhibitor of PI3k/Akt) at various concentrations $(0,5,10$, $20,40,80$ and $160 \mu \mathrm{M}$ ) for 24,48 and $72 \mathrm{~h}$. Cell proliferation and apoptosis were assessed by MTT and FACS assays, respectively. The migration and invasion abitility of the cells was investigated using wound healing and Transwell assays, respectively. In the MTT assays, the results revealed that LY294002 induced U2-OS cell apoptosis and inhibited cell growth in a dose- and time-dependent manner (Fig. 2A) and the $\mathrm{IC}_{50}$ was $47.96 \mu \mathrm{M}$ for $24 \mathrm{~h}$. The concentration of $40 \mu \mathrm{M}$ was selected for further experiments. Furthermore, in FACS assays, the results revealed that LY294002 induced U2-OS 


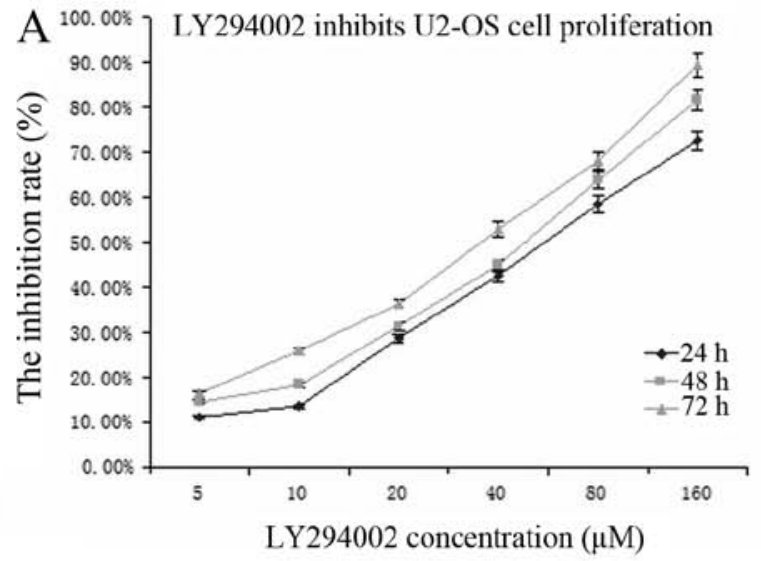

B

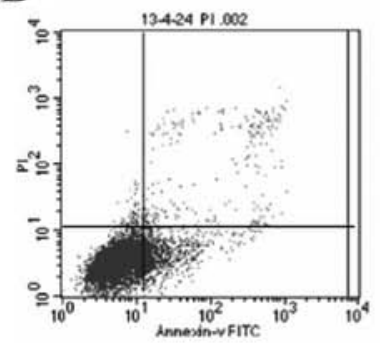

MR-Neg

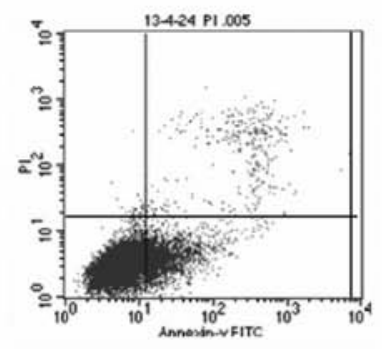

LY294002 $40 \mu \mathrm{M}$
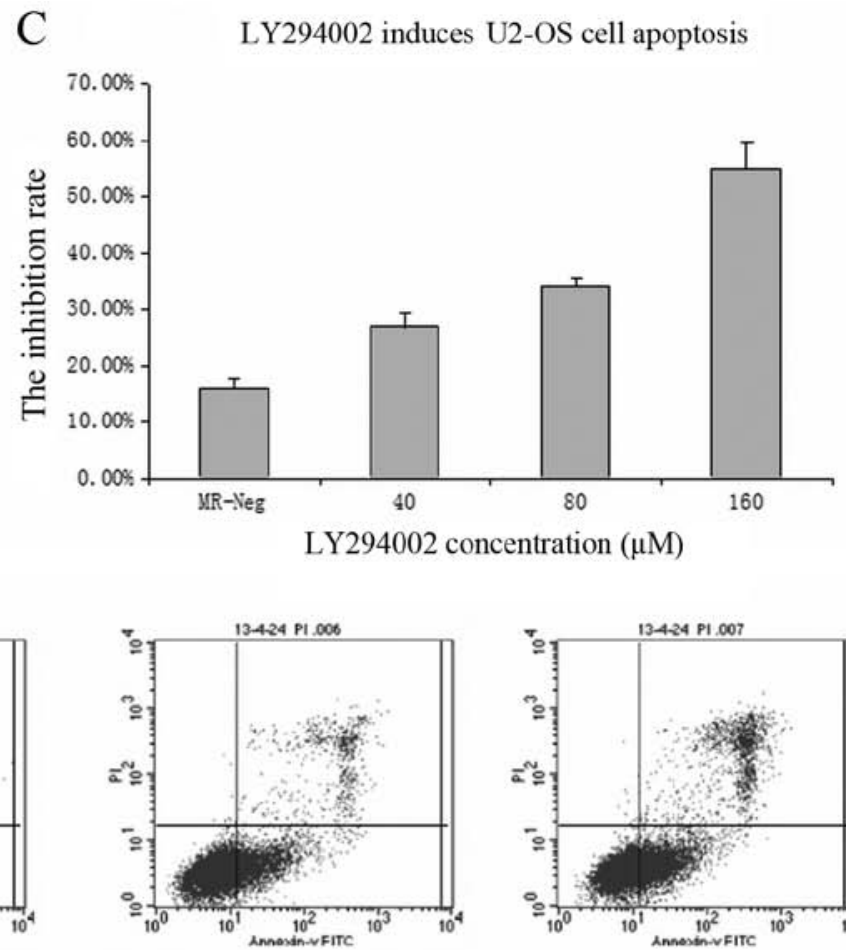

LY294002 $80 \mu \mathrm{M}$

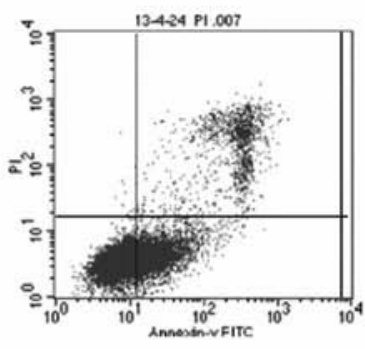

LY294002 $160 \mu \mathrm{M}$

Figure 2. Inhibition of Akt phosphorylation induces apoptosis and inhibits the growth of U2-OS cell. (A) The U2-OS cells were treated with LY294002 (an inhibitor of PI3K/Akt) at different concentrations for 24, 48 and $72 \mathrm{~h}$. LY294002 inhibited U2-OS cell proliferation in a dose- and time-dependent manner. (B and C) FACS analysis of U2-OS cells treated with LY294002 at different concentrations for $24 \mathrm{~h}$. LY294002 induced U2-OS cell apoptosis in a dose-dependent manner.
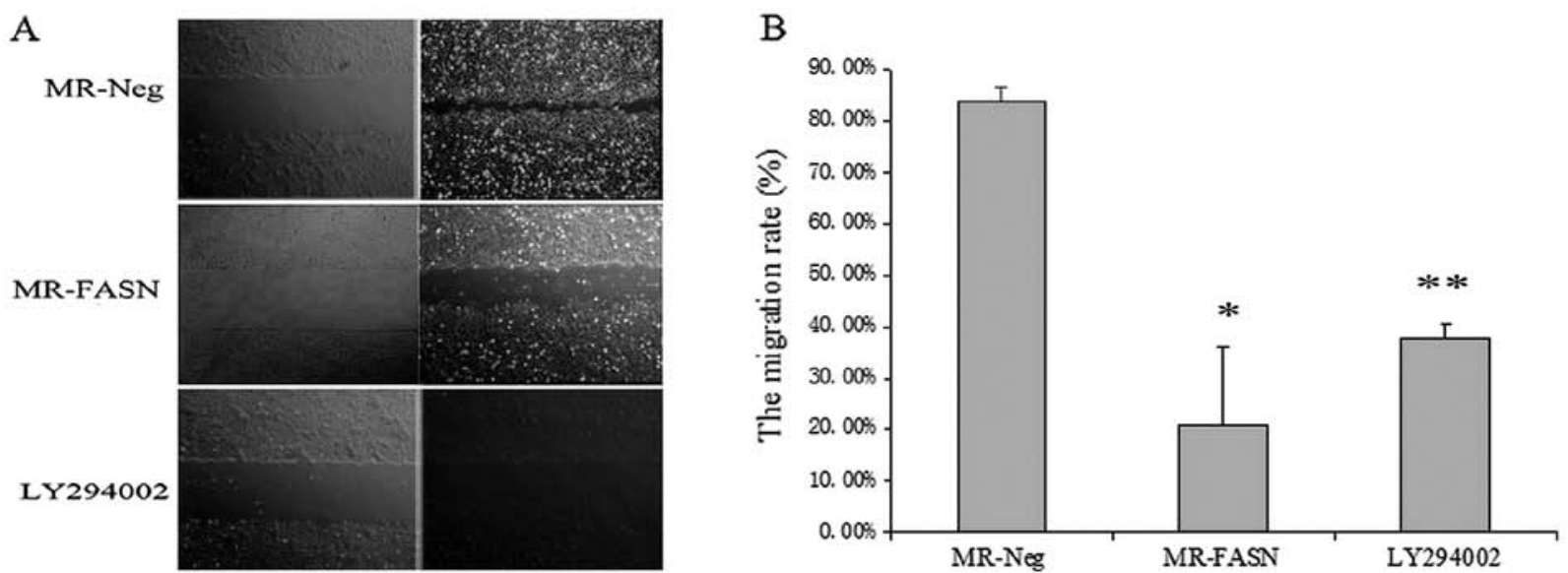

Figure 3. (A) Wound healing assay was performed to determine the effects of the downregulation of fatty acid synthase (FASN) or the inhibition of Akt phosphorylation on U2-OS cell migration. (B) Bars represent the means $+\mathrm{SD}(\mathrm{n}=6) ;{ }^{*, * *} \mathrm{P}<0.05$ vs. control group (MR-Neg).

cell apoptosis in a dose- and time-dependent manner (Fig. 2B and $\mathrm{C}$ ). In the the wound healing assay, the migration rate of the cells treated with $40 \mu \mathrm{M} \mathrm{LY} 294002$ was significantly lower than that of the control (untreated) cells $(\mathrm{P}<0.05)$ (Fig. 3). The percentage of invading cells in the group treated with the p-Akt inhibitor (LY294002) was 36.1 $\pm 5.0 \%$, compared with $89.1 \pm 6.4 \%$ in the control group $(\mathrm{P}<0.05)$ (Fig. 4).

Inhibition FASN suppresses U2-OS cell migration and invasion. In a previous study, we demonstrated that the suppression of FASN expression induces U2-OS cell apoptosis and inhibits cell growth in vivo and in vitro (17). Therefore, in this study, we evaluated the effects of the downregulation FASN on U2-OS cell migration and invasion. The migration and invasion ability of the cells was significantly lower in the cells transfected with the FASN-specific RNAi plasmid than those transfected with the negative RNAi plasmid $(\mathrm{P}<0.05)$ (Figs. 3 and 4).

Downregulation of FASN expression inhibits the phosphorylation of Akt. To investigate the effects of silencing FASN on the phosphorylation of Akt in OS, the U2-OS OS cells were transfected with the FASN-specific RNAi plasmid to inhibit 

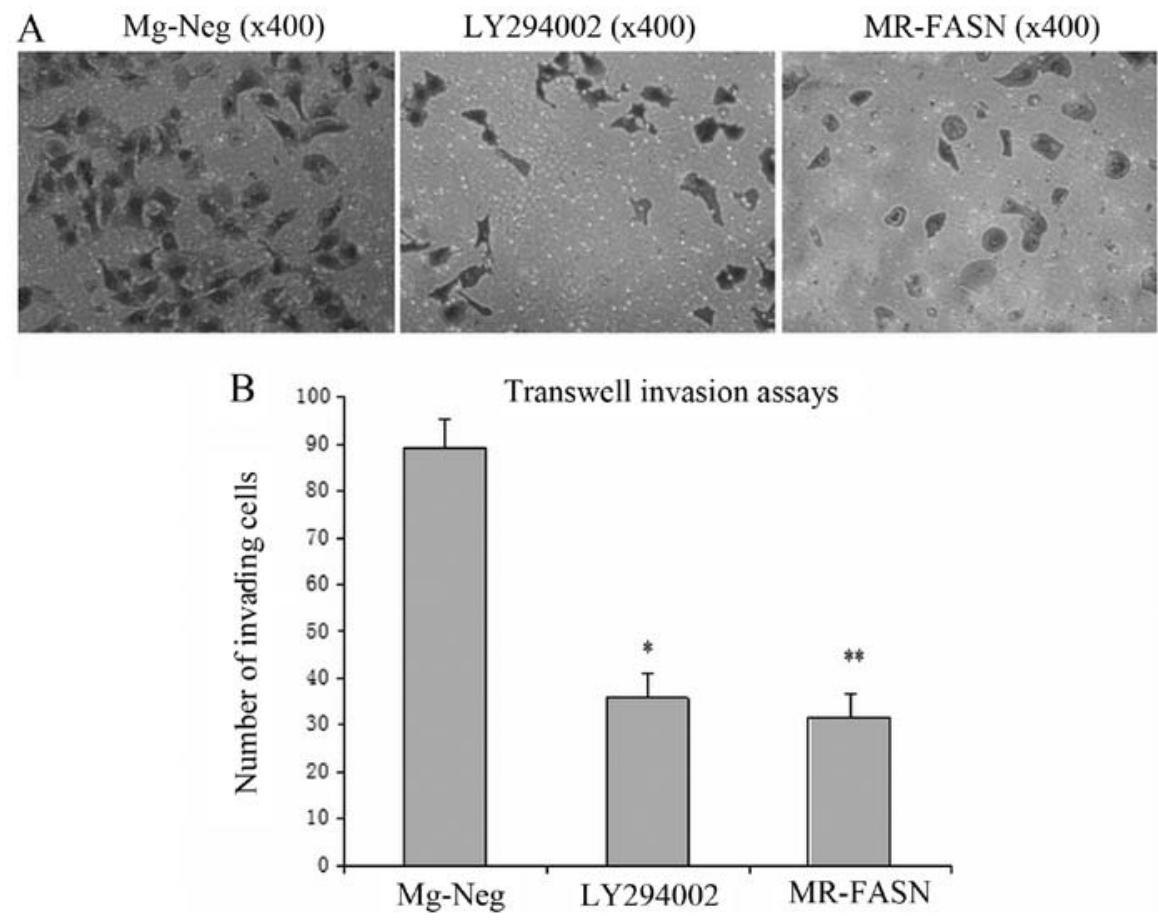

Figure 4. (A) Transwell invasion assay to evaluate the effects of the downregulation of fatty acid synthase (FASN) or the inhibition of Akt phosphorylation on U2-OS cell invasion. (B) Bars represent the means $+\mathrm{SD}(\mathrm{n}=6) ;{ }^{* * * *} \mathrm{P}<0.05$ vs. control group (MR-Neg).
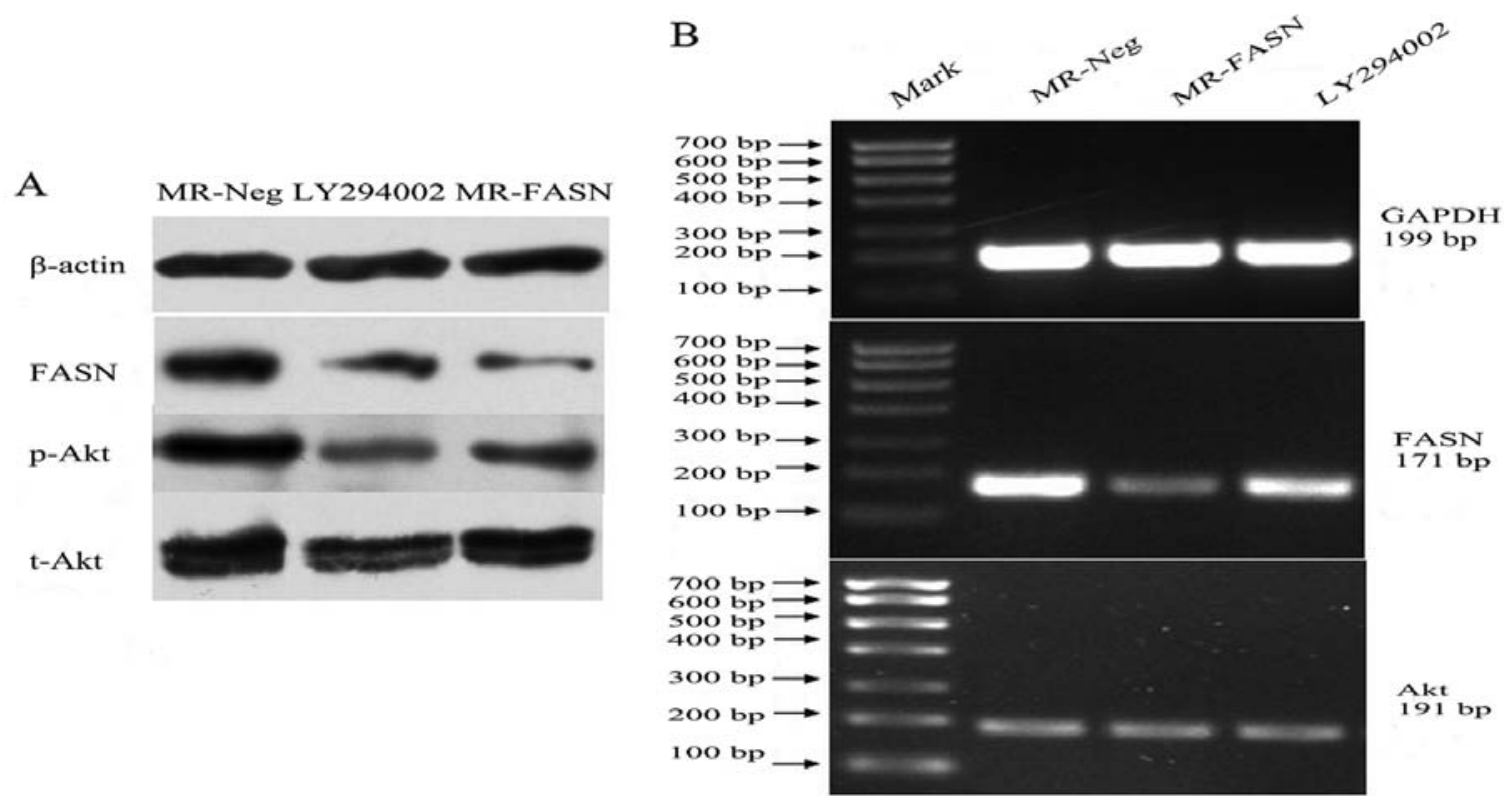

Figure 5. (A) Western blot analysis for the detection of the expression of phosphorylated Akt (p-Akt), Akt and fatty acid synthase (FASN) protein. The downregulation of of FASN inhibited Akt phosphorylation, and the inhibition of Akt phosphorylation downregulated FASN protein expression. (B) Real-time PCR was performed to measure the mRNA expression of Akt and FASN. The inhibition of Akt phosphorylation downregulated FASN mRNA expression; however, the downregulation of FASN had no significant inhibitory effect on Akt phosphorylation.

FASN expression. The mRNA expression of FASN and Akt was detected by real-time PCR, and western blot analysis was used to measure the protein expression of FASN, PI3K, Akt and p-Akt. The downregulation of FASN inhibited the activation of the PI3K/Akt signaling pathway. However, the mRNA expression of Akt was not affected by the downregulation of FASN in the U2-OS cells (Fig. 5A and B).
Inhibition of Akt phosphorylation downregulates FASN in U2-OS cells. In order to determine the effects of the inhibition of the phosphorylation of Akt on the mRNA and protein expression of FASN in OS, the U2-OS cells were treated with LY294002 (an inhibitor of PI3K/Akt). The mRNA and protein expression of FASN was measured by RT-PR and western blot analysis, respectively. The results revealed that both the 
mRNA and protein expression of FASN was decreased by the inhibition of Akt phosphorylation (Fig. 5).

\section{Discussion}

Previous studies have demonstrated that cancers with a high expression of FASN always undergo a significant endogenous fatty acid biosynthesis and display a biologically aggressive subset $(22,23)$. Moreover, the overexpression of FASN is an early event in tumor development and is more pronounced in tumors with a poor prognosis (24). Importantly, we previously demonstrated that the inhibition of FASN with pharmacological inhibitors is selectively cytotoxic to human OS cells and leads to a significant antitumor effect (17). Although very little is known about the mechanisms underlying the upregulation of the FAS protein in cancer cells, studies have revealed that FASN is also upregulated at the mRNA level $(25,26)$, and increasing evidence indicates that Akt activity modulates FASN expression in tumor cells $(20,27)$. Previous studies have revealed that activated $\mathrm{PI} 3 \mathrm{~K}$ stimulates the binding of sterol regulatory element-binding protein (SREBP)-1c, a SREBP family transcription factor, which controls genes involved in lipogenesis to a SREBP-binding site in the FASN promoter, thus inducing FASN transcription (28-30). Of note, the inhibition of FASN activity by either cerulenin or C75 has been shown to inhibit the prodution of p-Akt $(19,31)$. These findings suggest the existence of a positive bidirectional association between p-Akt and FASN expression in cancer cells.

In the current study, we found that there was a positive correlation between $\mathrm{p}-\mathrm{Akt}$ and FASN protein expression in OS tissues. This indicates that a possible connection between the phosphorylation of Akt and FASN expression may exist. In order to investigate whether the inhibition of the phosphorylation of Akt suppresses FASN expression in OS, LY294002, an inhibitor of PI3k/Akt, was used to downregulate the phosphorylation of Akt in U2-OS cells. The results revealed that the mRNA and protein expression of FASN was markedly inhibited by LY294002, which suggests that p-Akt regulates FASN by affecting the transcription and translation in U2-OS cells.

To determine the effects of the inhibition of FASN on the phosphorylation of Akt in OS cells, the U2-OS cells were transfected with the FASN-specific RNAi plasmid to inhibit FASN expression. We found that the protein expression of both total Akt and p-Akt was decreased in the FASN-silenced U2-OS cells. However, the mRNA expression of Akt did not differ between the cells treated with the FASN-specific RNAi plasmid and those treated with the negative RNAi plasmid. These data suggest that FASN regulates PI3K/Akt at the translational rather than the transcriptional level. However, the mechanism(s) responsible for the inhibition of Akt activity by the downregulation of FASN remain unclear. Currently, several mechanisms are likely to contribute to FASN-mediated p-Akt regulation: i) FASN-mediated lipogenesis produces phospholipids that are incorporated into cell membranes and partition into lipid rafts, which accommodate ErbBs and form signaling platforms. FASN blockade destabilizes these lipid rafts, which triggers the degradation of ErbBs and impedes the membrane recruitment of downstream mediators of Akt, thereby causing the downregulation of p-Akt $(32,33)$; ii) the FASN promoter contains several sterol regulatory element-binding protein-1 (Sp1) sites enabling FASN to activate Akt $(34,35)$.

Recently, a number of studies have demonstrated that FASN and PI3K/Akt play an important role in the proliferation, invasion and migration of cancer cells (36-39). In this study, we observed that the suppression of FASN expression by RNAi or the inhibition of the activity of PI3K/Akt blocked cell proliferation and migration and increased the apoptotic rate in the U2-OS cells. This suggests that FASN and PI3K/Akt play a key role in the maintenance of the malignant phenotype of OS cells.

In conclusion, the data presented in this study, confirm the existence of a positive feedback loop between Akt phosphorylation and FASN expression. Moreover, this feedback loop plays an important role in the malignant phenotype of OS cells. However, the detailed mechanisms of the bidirectional association between FASN expression and the phosphorylation of Akt in OS cells are currently unknown. Thus, further studies are required to provide further clarification.

\section{Acknowledgements}

The present study was supported by grants from the National Natural Science Foundation of China (no. 81260400) and the Natural Science Fundation of Jiangxi Province (no. 20114BAB205093).

\section{References}

1. Meyers PA, Schwartz CL, Krailo M, Kleinerman ES, Betcher D, Bernstein ML, et al: Osteosarcoma: a randomized, prospective trial of the addition of ifosfamide and/or muramyl tripeptide to cisplatin, doxorubicin, and high-dose methotrexate. J Clin Oncol 23: 2004-2011: 2005.

2. Bacci G, Forni C, Longhi A, Ferrari S, Mercuri M, Bertoni F, et al: Local recurrence and local control of non-metastatic osteosarcoma of the extremities: a 27-year experience in a single institution. J Surg Oncol 96: 118-123, 2007.

3. Jawad MU, Cheung MC, Clarke J, Koniaris LG and Scully SP: Osteosarcoma: improvement in survival limited to high-grade patients only. J Cancer Res Clin Oncol 137: 597-607, 2011.

4. Mialou V, Philip T, Kalifa C, Perol D, Gentet JC, MarecBerard $\mathrm{P}$, et al: Metastatic osteosarcoma at diagnosis: prognostic factors and long-term outcome--the French pediatric experience. Cancer 04: 1100-1109, 2005.

5. Hegyi M, Semsei AF, Jakab Z, Antal I, Kiss J, Szendroi M, et al: Good prognosis of localized osteosarcoma in young patients treated with limb-salvage surgery and chemotherapy. Pediatr Blood Cancer 57: 415-22, 2011.

6. Stokkel MP, Linthorst MF, Borm JJ, Taminiau AH and Pauwels EK: A reassessment of bone scintigraphy and commonly tested pretreatment biochemical parameters in newly diagnosed osteosarcoma. J Cancer Res Clin Oncol 128: 393-399, 2002.

7. Kuhajda FP: Fatty acid synthase and cancer: new application of an old pathway. Cancer Res 66: 5977-5980, 2006.

8. Alo PL, Amini M, Piro F, Pizzuti L, Sebastiani V, Botti C, et al: Immunohistochemical expression and prognostic significance of fatty acid synthase in pancreatic carcinoma. Anticancer Res 27: 2523-2527, 2007

9. Walter K, Hong SM, Nyhan S, Canto M, Fedarko N, Klein A, et al: Serum fatty acid synthase as a marker of pancreatic neoplasia. Cancer Epidemiol Biomarkers Prev 18: 2380-2385, 2009.

10. Okawa Y, Hideshima T, Ikeda H, Raje N, Vallet S, Kiziltepe T, et al: Fatty acid synthase is a novel therapeutic target in multiple myeloma. Br J Haemato 141: 659-671, 2008.

11. Migita T, Ruiz S, Fornari A, Fiorentino M, Priolo C, Zadra G, et al: Fatty acid synthase: a metabolic enzyme and candidate oncogene in prostate cancer. J Natl Cancer Inst 101: 519-532, 2009. 
12. Silva SD, Cunha IW, Younes RN, Soares FA, Kowalski LP and Graner E: ErbB receptors and fatty acid synthase expression in aggressive head and neck squamous cell carcinomas. Oral Dis 16: 774-780, 2010

13. Saati GE and Archer MC: Inhibition of fatty acid synthase and Sp1 expression by 3,3'-diindolylmethane in human breast cancer cells. Nutr Cancer 63: 790-794, 2011.

14. Notarnicola M, Pisanti S, Tutino V, Bocale D, Rotelli MT, Gentile A, et al: Effects of olive oil polyphenols on fatty acid synthase gene expression and activity in human colorectal cancer cells. Genes Nutr 6: 63-69, 2011.

15. Notarnicola M, Messa C, Refolo MG, Tutino V, Miccolis A and Caruso MG: Polyunsaturated fatty acids reduce fatty acid synthase and hydroxy-methyl-glutaryl CoA-reductase gene expression and promote apoptosis in HepG2 cell line. Lipids Health Dis 10: 10, 2011.

16. Zecchin KG, Rossato FA, Raposo HF, Melo DR, Alberici LC Oliveira HC, et al: Inhibition of fatty acid synthase in melanoma cells activates the intrinsic pathway of apoptosis. Lab Invest 91: 232-240, 2011.

17. Long XH, Mao JH, Peng AF, Zhou Y, Huang SH and Liu ZL: Tumor suppressive microRNA-424 inhibits osteosarcoma cell migration and invasion via targeting fatty acid synthase. Exp Ther Med 5: 1048-1052, 2013.

18. Liu ZL, Wang G, Peng AF, Luo QF, Zhou Y and Huang SH: Fatty acid synthase expression in osteosarcoma and its correlation with pulmonary metastasis. Oncol Lett 4: 878-882, 2012.

19. Liu X, Shi Y, Giranda VL and Luo Y: Inhibition of the phosphatidylinositol 3-kinase/Akt pathway sensitizes MDA-MB468 human breast cancer cells to cerulenin-induced apoptosis. Mol Cancer Ther 5: 494-501, 2006.

20. Van de Sande T, De Schrijver E, Heyns W, Verhoeven G and Swinnen JV: Role of the phosphatidylinositol 3'-kinase/PTEN/Akt kinase pathway in the overexpression of fatty acid synthase in LNCaP prostate cancer cells. Cancer Res 62: 642-646, 2002.

21. Wang HQ, Altomare DA, Skele KL, Poulikakos PI, Kuhajda FP, Di Cristofano A, et al: Positive feedback regulation between AKT activation and fatty acid synthase expression in ovarian carcinoma cells. Oncogene 24: 3574-3582, 2005.

22. Menendez JA and Lupu R: Fatty acid synthase-catalyzed de novo fatty acid biosynthesis: from anabolic-energy-storage pathway in normal tissues to jack-of-all-trades in cancer cells. Arch Immunol Ther Exp (Warsz) 52: 414-426, 2004.

23. Menendez JA, Ropero S, Mehmi I, Atlas E, Colomer R and Lupu R: Overexpression and hyperactivity of breast cancerassociated fatty acid synthase (oncogenic antigen-519) is insensitive to normal arachidonic fatty acid-induced suppression in lipogenic tissues but it is selectively inhibited by tumoricidal $\alpha$-linolenic and gamma-linolenic fatty acids: A novel mechanism by which dietary fat can alter mammary tumorigenesis. Int J Oncol 24: 1369-1383, 2004.

24. Porter D, Lahti-Domenici J, Keshaviah A, Bae YK, Argani P, Marks J, et al: Molecular markers in ductal carcinoma in situ of the breast. Mol Cancer Res: 362-375, 2003.

25. Swinnen JV, Vanderhoydonc F, Elgamal AA, Eelen M, Vercaeren I, Joniau S, et al: Selective activation of the fatty acid synthesis pathway in human prostate cancer. Int J Cancer 88 : $176-179,2000$
26. Milgraum LZ, Witters LA, Pasternack GR and Kuhajda FP. Enzymes of the fatty acid synthesis pathway are highly expressed in in situ breast carcinoma. Clin Cancer Res 3: 2115-2120, 1997.

27. Yeh CW, Chen WJ, Chiang CT, Lin-Shiau SY and Lin JK: Suppression of fatty acid synthase in MCF-7 breast cancer cells by tea and tea polyphenols: a possible mechanism for their hypolipidemic effects. Pharmacogenomics J 3: 267-276, 2003.

28. Bandyopadhyay S, Pai SK, Watabe M, Gross SC, Hirota S, Hosobe S, et al: FAS expression inversely correlates with PTEN level in prostate cancer and a PI 3-kinase inhibitor synergizes with FAS siRNA to induce apoptosis. Oncogene 24: 5389-5395, 2005.

29. Swinnen JV, Heemers H, Deboel L, Foufelle F, Heyns W and Verhoeven G: Stimulation of tumor-associated fatty acid synthase expression by growth factor activation of the sterol regulatory element-binding protein pathway. Oncogene 19: 5173-81, 2000.

30. Yang Y, Morin PJ, Han WF, Chen T, Bornman DM, Gabrielson EW and Pizer ES: Regulation of fatty acid synthase expression in breast cancer by sterol regulatory element binding protein-1c. Exp Cell Res 282: 132-137, 2003.

31. Alli PM, Pinn ML, Jaffee EM, McFadden JM and Kuhajda FP: Fatty acid synthase inhibitors are chemopreventive for mammary cancer in neu-N transgenic mice. Oncogene 24: 39-46, 2005.

32. Scheid MP, Marignani PA and Woodgett JR: Multiple phosphoinositide 3-kinase-dependent steps in activation of protein kinase B. Mol Cell Biol 22: 6247-6260, 2002.

33. Li N, Lu H, Chen C, Bu X and Huang P: Loss of fatty acid synthase inhibits the 'HER2-PI3K/Akt axis' activity and malignant phenotype of Caco-2 cells. Lipids Health Dis 12: 83, 2013.

34. Jin HO, An S, Lee HC, Woo SH, Seo SK, Choe TB, et al: Hypoxic condition- and high cell density-induced expression of Redd1 is regulated by activation of hypoxia-inducible factor-1alpha and Sp1 through the phosphatidylinositol 3-kinase/Akt signaling pathway. Cell Signal 19: 1393-1403, 2007.

35. Roder K, Wolf SS, Larkin KJ and Schweizer M: Interaction between the two ubiquitously expressed transcription factors NF-Y and Sp1. Gene 234: 61-69, 1999.

36. Deepa PR, Vandhana S and Krishnakumar S: Fatty acid synthase inhibition induces differential expression of genes involved in apoptosis and cell proliferation in ocular cancer cells. Nutr Cancer 65: 311-316, 2013.

37. Rahman MT, Nakayama K, Ishikawa M, Rahman M, Katagiri H, Katagiri A, et al: Fatty acid synthase is a potential therapeutic target in estrogen receptor-/progesterone receptor-positive endometrioid endometrial cancer. Oncology 84: 166-173, 2013.

38. Zhou JD, Shen F, Ji JS, Zheng K, Huang M and Wu JC: FAM9C plays an anti-apoptotic role through activation of the PI3K/Akt pathway in human hepatocellular carcinoma. Oncol Rep 2013: doi: $10.3892 /$ or.2013.2592.

39. Yothaisong S, Dokduang H, Techasen A, Namwat N, Yongvanit P, Bhudhisawasdi $\mathrm{V}$, et al: Increased activation of PI3K/AKT signaling pathway is associated with cholangiocarcinoma metastasis and PI3K/mTOR inhibition presents a possible therapeutic strategy. Tumour Biol 34: 3637-3648, 2013. 